\title{
FADIGA NA PESSOA COM SEQUELAS DA COVID-19, UMA PROPOSTA DE REABILITAÇÃO: ESTUDO DE CASO
}

\author{
FATIGUE IN PERSON WITH SEQUELS OF COVID-19, A PROPOSAL FOR REHABILITATION: CASE STUDY
}

FATIGA EN PERSONA CON SECUELAS DE COVID-19, UNA PROPUESTA DE REHABILITACIÓN: ESTUDIO DE CASO

DOI 10.33194/rper.2022.185

Data de Receção: 2021-09-14 Data de Aceitação: 2022-01-01 Data de publicação on-line: 2022-01-19

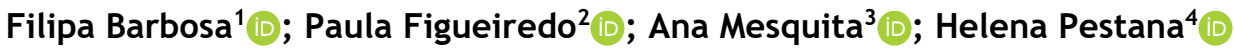 \\ ${ }^{1}$ Hospital CUF Tejo; ${ }^{2}$ CHULC; Hospital do Mar Cuidados Especializados; ${ }^{3}$ Escola Superior de Saúde Atlântica; \\ ${ }^{4} \mathrm{CHULC}$, Escola Superior de Saúde Atlântica
}

Autor correspondente: Helena Pestana, hcpestana@gmail.com

\section{RESUMO}

Introdução: A COVID-19 pode causar sequelas persistentes, sendo a mais descrita a fadiga. Os enfermeiros especialistas em enfermagem de reabilitação são portadores de conhecimento especializado no controlo e redução da mesma.

Objetivo: Pretende-se identificar os ganhos sensíveis à reabilitação respiratória no foco de ventilação e intolerância à atividade; analisar a tolerância ao esforço na pessoa após infeção por COVID-19 e verificar a efetividade das intervenções do enfermeiro especialista em enfermagem de reabilitação no controlo e redução da fadiga em pessoas com sequelas de COVID-19.

Método: Estudo de caso com recolha de dados quantitativos e qualitativos. Apresenta-se o caso de uma pessoa com sequelas pós-COVID e fraqueza muscular adquirida nos cuidados intensivos, caracterizada por uma ventilação ineficaz e intolerância à atividade.

Resultados: 0 processo de enfermagem, integrou as recomendações de reabilitação respiratória da Sociedade Chinesa de Medicina de Reabilitação, com 14 sessões verificou-se: redução de fadiga, aumento da capacidade vital, aumento das distâncias percorridas e aumento da autonomia na realização das AVD.

Conclusões: Com a implementação de um plano de cuidados de enfermagem de reabilitação individualizado é possível atuar no controlo e redução da fadiga em pessoas com sequelas da COVID-19, proporcionando ganhos em saúde sensíveis aos cuidados aos cuidados de enfermagem de reabilitação.

DESCRITORES: Fadiga, Reabilitação Respiratória, COVID-19, Enfermagem de Reabilitação; Intolerância à atividade.

\section{ABSTRACT}

Background: COVID-19 can cause persistent sequelae, being fatigue the most described. Specialist nurses in rehabilitation nursing have specialized knowledge in the control and reduction of fatigue.

Objective: It is intended to identify the gains sensitive to respiratory rehabilitation in the focus of ventilation and activity intolerance; analyze the effort tolerance in the person after infection with-COVID-19 and verify the effectiveness of the interventions of the specialist nurse in rehabilitation nursing in the control and reduction of fatigue in people with sequelae of COVID-19.

Method: Case study with quantitative and qualitative data collection. We present the case of a person with postCOVID sequelae and muscle weakness acquired in intensive care, characterized by ineffective ventilation and activity intolerance.

Results: The nursing process, integrated the respiratory rehabilitation recommendations of the Chinese Society of Rehabilitation Medicine. With 14 rehabilitation sessions, it was found: reduced fatigue, increased vital capacity, and increased distances covered and increased autonomy in performing ADLs.

Conclusions: With the implementation of an individualized rehabilitation nursing care plan, it is possible to act in the control and reduction of fatigue in people with sequelae of COVID-19, providing health gains that are sensitive to the care of rehabilitation nursing care. 
DESCRIPTORS: Fatigue, Respiratory Rehabilitation, COVID-19, Rehabilitation Nursing; Activity intolerance.

\section{RESUMEN}

Introducción: COVID-19 puede causar secuelas persistentes, siendo la fatiga la más descrita. Los enfermeros especialistas en rehabilitación de enfermería cuentan con conocimientos especializados en el control y reducción de la fatiga.

Objetivo: Se pretende identificar las ganancias sensibles a la rehabilitación respiratoria en el foco de la ventilación y la intolerancia a la actividad; analizar la tolerancia al esfuerzo en la persona después de la infección por COVID19 y verificar la efectividad de las intervenciones de la enfermera especialista en rehabilitación de enfermería en el control y reducción de la fatiga en personas con secuelas de COVID-19.

Método: Estudio de caso con recopilación de datos cuantitativos y cualitativos. Presentamos el caso de una persona con secuelas post-COVID y debilidad muscular adquirida en cuidados intensivos, caracterizada por ventilación ineficaz e intolerancia a la actividad.

Resultados: Utilizando el proceso de enfermería integrando las recomendaciones de rehabilitación respiratoria de la Sociedad China de Medicina de Rehabilitación, con 14 sesiones de rehabilitación se encontró: reducción de fatiga, aumento de capacidad vital, aumento de las distancias recorridas y aumento de la autonomía en la realización de la ADL.

Conclusiones: Con la implementación de un plan de atención de enfermería rehabilitadora individualizada, es posible actuar en el control y reducción de la fatiga en personas con secuelas de COVID-19, brindando ganancias en salud sensibles al cuidado de la enfermería rehabilitadora.

DESCRIPTORES: Fatiga, Rehabilitación respiratoria, COVID-19, Enfermería de rehabilitación; Intolerancia a la actividad.

\section{INTRODUÇÃO}

A atual pandemia causada pelo vírus SARS-CoV-2 é um dos maiores desafios dos sistemas de saúde nos dias de hoje, tendo-se disseminado rapidamente por todo o mundo condicionando no futuro um conjunto de sequelas cujo impacto ainda está em avaliação. Em Portugal, o primeiro caso surgiu a 1 de março de 2020, mês em que a Organização Mundial de Saúde (OMS) declarou a COVID-19 uma pandemia global e emergência de saúde pública ${ }^{1,2}$.

O SARS-CoV-2 sabe-se hoje que é uma variação genética de uma estripe de coronavírus designado por síndrome respiratória aguda grave e que possui um elevado risco de disseminação humano-humano através da inalação de gotículas respiratórias produzidas, pela tosse, espirro ou fala, e/ou contacto das mãos com uma superfície contaminada ${ }^{1,3,4}$. A doença tem um período de incubação e contágio de 3 a 7 dias, até um máximo de 14 dias para aparecimento de sintomas ${ }^{1,3,5}$, sendo consensual entre os diversos estudos as suas manifestações clínicas.

A doença envolve as vias respiratórias inferiores, e condiciona sintomas dominantes como: febre, tosse seca, fadiga, mialgias e dispneia ${ }^{1,6,7}$, bem como, fervores crepitantes e diminuição do murmúrio vesicular à auscultação pulmonar, e hiporressonância à percussão pulmonar. 0 diagnóstico pode ser confirmado pela existência de teste de reação em cadeia de transcriptase reversa (RT-PCR) positivo, e as alterações em exames radiológicos desempenham a função de diagnóstico presuntivo. Estudos demonstram o predomínio de opacidades em vidro despolido, bronquiectasias, espessamento pleural e pneumonia organizativa na sua maioria bilateral, sendo a ausência de derrame pleural e de adenopatias característico desta patologia ${ }^{8}$.

Com risco acrescido de mau prognóstico para a população com mais de 60 anos, doentes imunossuprimidos e doentes $\operatorname{crónicos}^{3,6}$, as formas de apresentação da doença podem ser ligeiras a moderadas caracterizando-se por doença respiratória ligeira sem insuficiência respiratória até pneumonia extensa hipoxemiante ${ }^{3}$. Os números publicados em Portugal refletem que em média, $20 \%$ dos casos graves necessitam de internamento sendo $10 \%$ desses considerados críticos com necessidade de cuidados intensivos (UCl) ${ }^{5}$.

Assim, à semelhança de outras pneumonias virais, o tratamento contra a COVID-19 baseia-se no controlo sintomático e terapêutica de suporte, como controlo de temperatura e sintomas respiratórios (casos ligeiros), oxigénio nasal de alto fluxo ou ventilação mecânica não invasiva (casos graves) e ventilação mecânica invasiva, oxigênio por membrana extracorpórea (ECMO) ${ }^{1,9}$ e técnica de substituição renal (casos críticos).

Um ano depois, com o aumento do número de casos recuperados denotou-se que após a alta hospitalar os doentes apresentam défices no autocuidado ${ }^{10,11}$. As sequelas, têm mostrado afetar predominantemente os sistemas respiratório, cardiovascular, renal e neurológico, com sintomas persistentes de dispneia, fadiga, tosse, toracalgia, mialgia, cefaleias, palpitações, anosmia, hiposmia, anorexia e distúrbios cognitivos ${ }^{2,10,11,12}$ que caracterizam a 
síndrome pós-covid. Os sintomas podem persistir durante mais de 3 semanas em $10 \%$ dos casos ${ }^{12}$, mas é reportada igualmente a sua persistência até 60 a 110 dias após a alta hospitalar com incidência elevada de fadiga como sintoma mais comum, significativo e duradouro $2,10,12,13$. A experiência tem demonstrado que independentemente da gravidade da doença, hospitalização ou sob isolamento domiciliário, a não recuperação total após 2 a 3 semanas da testagem positiva foi relatada por aproximadamente um terço das pessoas ${ }^{14,15}$.

Com o crescente número de doentes com síndrome pós-COVID, a reabilitação constitui um componente-chave da recuperação. Sendo a disciplina que visa melhorar a funcionalidade, espera-se do EEER a implementação de medidas não farmacológicas que promovam a recuperação da independência nas atividades de vida diária (AVD) com vista à diminuição do risco de morbilidade e à recuperação a médio/ longo prazo ${ }^{3,7}$. Em Portugal a sua intervenção é reconhecida, iniciando-se em meio hospitalar com intenção de redução do tempo de internamento e progressão para cuidados de reabilitação no domiciliário ${ }^{5}$.

A pneumonia por COVID-19 é uma doença cujo dano pulmonar é causado por infiltrado intersticial pulmonar bilateral, comprometendo a razão ventilação/ perfusão, a complacência pulmonar e a uma elevada recrutabilidade alveolar. 0 exsudado alveolar provocado pelo processo cicatricial inflamatório pode dar origem a fibrose pulmonar que afeta a função pulmonar de $45 \%$ dos doentes no prazo de 1 mês após infeção ou desenvolve-se entre 3 a 6 meses após ${ }^{9,15}$. Além disso, também podem ocorrer sequelas de envolvimento vascular com consequente trombose venosa, miocardite e arritmias; insuficiência hepática e renal, sarcopenia aguda e acidente vascular cerebral 3,9,16, que contribuem para diminuição da capacidade funcional.

Tendo em conta os compromissos provocados pelo internamento em $\mathrm{UCl}$, estima-se que os défices provocados pela FMACI (Fraqueza Muscular Associada aos Cuidados Intensivos), possam persistir por meses ou até anos após doença, com elevada causa-efeito na força muscular, musculatura respiratória, tónus muscular, amplitude articular, deglutição, equilibro e coordenação, e com desfecho desfavorável na qualidade de vida e realização das AVD ${ }^{3,15,16}$.

A fadiga e a intolerância ao exercício são os sintomas mais comuns a longo prazo. As causas de fadiga, podem ser atribuídas ao declínio da tolerância ao exercício associado a disfunção cardiopulmonar, perda de função ventilatória persistente e atrofia muscular ${ }^{4}$. Estes sintomas, além de avaliados subjetivamente pela pessoa através de instrumentos de avaliação validados, com recurso ao exame físico, avaliação da capacidade funcional, da função respiratória e muscular, e da qualidade de vida ${ }^{16}$ com o objetivo de elaborar um plano de Reabilitação Respiratória (RR) individualizado. Neste estudo iremos recorrer a avaliação da fadiga através da perceção subjetiva do esforço com recurso à escala de BORG.

Assim, o Enfermeiro Especialista em Enfermagem de Reabilitação (EEER) apresenta um papel multifatorial na reabilitação da pessoa com sintomatologia persistente pós-covid. Cabe-lhe estruturar um plano de reabilitação, com base nos princípios do método $4 \mathrm{~S}$ "simple, safe, satisfy, save"7, com recurso a técnicas específicas para melhorar a função respiratória, neutralizar a imobilidade, reduzir a taxa de complicações e de incapacidade a longo prazo, e melhorar os domínios cognitivos e emocionais ${ }^{5}$. Técnicas como exercícios respiratórios com inspiração profunda e sustentada associada a expiração com resistência, exercícios de relaxamento, exercícios abdominodiafragmáticos, exercícios de expansão torácica, treino dos músculos respiratórios, exercícios de fortalecimento muscular ${ }^{4,5,7}$, técnicas de conservação de energia ${ }^{15}$, treino aeróbio, treino de tosse ${ }^{18}$, treino de equilíbrio dinâmico ${ }^{3}$, estão documentadas pela sua eficácia na melhoria da função respiratória, capacidade física e funcional, determinante para o retorno às AVD.

O presente estudo de caso tem como questão norteadora "Qual o contributo do Enfermeiro Especialista em Enfermagem de Reabilitação no controlo e redução da fadiga apresentada por pessoas com sequelas de COVID-19?”. Tendo em conta os objetivos:

- Identificar os ganhos sensíveis à reabilitação respiratória no foco ventilação e intolerância à atividade;

- Analisar a tolerância ao esforço na pessoa após infeção por COVID-19;

- Verificar a efetividade das intervenções do EEER no controlo e redução da fadiga em pessoas com sequelas de COVID-19.

\section{METODOLOGIA}

Constitui um estudo exploratório do tipo estudo de caso com extração de dados quantitativos e qualitativos que visa evidenciar os benefícios da implementação de um plano de RR no controlo e redução da fadiga na pessoa em fase subaguda da COVID-19, em regime de internamento de convalescença em unidade de reabilitação, integrando dados obtidos no processo assistencial com os dados de evidência científica ${ }^{19}$.

O estudo foi elaborado a partir de um método de pesquisa com investigação empírica direcionada para o contexto da prática clínica, através da pesquisa em bases de dados bibliográficas eletrónicas como: SciELO, RCAAP, PubMed, Medline e CINAHL.

Como prática reflexiva teve o referencial teórico de Afaf Meleis - a Teoria das Transições. Este modelo conceptual, permitiu melhorar a experiência da pessoa durante o processo de transição, através da criação de elos facilitadores 
entre a rotura e o processo de mudança, com o objetivo de maximizar o potencial de conhecimentos e capacidades $^{20}$.

O consentimento informado foi assinado pelo próprio, tendo sido seguidas as diretrizes da ética para a investigação em enfermagem e a pessoa informada dos seus direitos na participação no estudo, respeitando o princípio da dignidade e da privacidade, bem como da não maleficência, vulnerabilidade, veracidade e confidencialidade.

À data, a China é o pais com maior número de casos recuperados no período de um ano, por consequente, a experiência acumulada e a evidência científica na área da reabilitação encontra-se mais aprofundada. Atendendo a que se trata de uma patologia recente e à rápida evolução do conhecimento científico nesta área, no que diz respeito a indicaç̃os/guidelines, o programa instituído foi apoiado nas diretrizes da Sociedade Chinesa de Medicina de Reabilitação ${ }^{21,22}$ e da Sociedade Portuguesa de Pneumologia ${ }^{23}$ e nas bases teóricas da enfermagem de reabilitação: Guia Orientador da Boa Prática para a Reabilitação Respiratória ${ }^{17}$.

A colheita de dados foi realizada através: da anamnese, da consulta do processo clínico e da prestação direta de cuidados.

Tendo por base que se trata de uma patologia restritiva, caracterizada por padrão respiratório ineficaz, considerouse como componentes da avaliação: o exame físico (inspeção e auscultação); a perceção subjetiva ao esforço através da escala de Borg Modificada; a distância percorrida no teste de marcha de 6 minutos (TM6) e a capacidade vital pulmonar (CV) avaliada por inspirometria de incentivo.

Tabela 1 - Escala de Borg Modificada

\begin{tabular}{|c|c|}
\hline 0 & Absolutamente nada \\
\hline 1 & Muito Pouca \\
\hline 2 & Pouca \\
\hline 3 & Média, Regular \\
\hline 4 & Um pouco forte \\
\hline 5 & Forte \\
\hline 6 & \\
\hline 7 & Muito forte \\
\hline 8 & \\
\hline 9 & Fortíssima \\
\hline 10 & Máxima \\
\hline
\end{tabular}

A escala de Borg Modificada (tabela 1) serviu que preditor na prescrição e ajuste do exercício. Compreendendo uma avaliação numérica de 0 a 10 , sendo que 0 corresponde à ausência de dispneia e 10 à sensação máxima de dispneia ${ }^{17}$, esta foi avaliada sempre antes, durante e após sessão de RR e TM6.

O TM6 é um teste submáximo recomendado para avaliação da capacidade ao esforço em pessoas com patologia respiratória. Implica a medição da distância percorrida durante 6 minutos, num percurso de 30 metros, tendo sido realizado após aquisição de equilíbrio dinâmico em ortostatismo e um mínimo de 10 minutos de repouso.

O inspirómetro de incentivo é um dispositivo que requer a execução de inspirações lentas e profundas com o objetivo de aumentar os volumes inspiratórios e melhorar o desempenho dos músculos inspiratórios.

$\mathrm{Na}$ abordagem à pessoa foram aplicadas as medidas de proteção individual definidas pela Direção Geral de Saúde de acordo com a Norma $n^{\circ}$ 007/2020 “Prevenção e Controlo de Infeção por SARS-CoV-2 (COVID-19): Equipamentos de Proteção Individual (EPI)"24 e as orientações sobre cuidados para pessoas com COVID-19 publicadas pela Mesa do Colégio da Especialidade de Enfermagem de Reabilitação ${ }^{5}$.

Os critérios de seleção basearam-se num único critério de inclusão: a existência de disfunções físicas, cognitivos ou mentais após alta hospitalar, em pessoas com internamento prévio por COVID-19.

Para garantir a segurança da pessoa, foram definidos critérios limitadores para início da sessão e normas para suspensão do exercício ${ }^{(21)}$ durante a sessão explicitados na tabela 2 e 3 .

Tabela 2 - Contraindicações relativas para início da sessão

Frequência cardíaca > 100 batimentos/ min

Pressão arterial < 90/60 mmHg ou > of $140 / 90 \mathrm{mmHg}$

Saturação de oxigênio no sangue, em repouso $\leq 95 \%$

Fonte: Sociedade Chinesa de Medicina de Reabilitação
Tabela 3 - Critérios de interrupção do exercício

Agravamento dos sintomas respiratórios ou da fadiga, sem alívio após o repouso

Aparecimento de um ou mais dos seguintes sintomas: aperto ou dor no peito, dispneia, tosse severa, tontura, cefaleia, visão turva, palpitações, sudorese profusa e marcha instável.

Fonte: Sociedade Chinesa de Medicina de Reabilitação 


\section{APRESENTAÇÃO DO CASO}

Trata-se uma pessoa de 70 anos, do género feminino. Previamente autónoma para todas as AVD. Tem como antecedentes pessoais conhecidos: Hipotiroidismo, Dislipidemia, Hipertensão arterial, Tabagismo, Lente intraocular bilateral, Doença osteoarticular degenerativa, Bronquiectasias.

Recorreu ao serviço de urgência em 09/2020 por quadro de dispneia com tosse produtiva, edemas dos membros inferiores e nictúria. 8 dias depois é-lhe diagnosticada pneumonia a SARS-CoV-2 com sobreinfecção bacteriana e é transferida para $\mathrm{UCl}$, inicialmente sob ventilação mecânica não invasiva acabando por ser submetida a ventilação invasiva 24 horas depois. Durante a permanência em UCI houve progressão do quadro de insuficiência respiratória, com diversas intercorrências infeciosas, pneumotórax espontâneo, delírio e $\mathrm{FMACl}$ com necessidade de traqueostomia cirúrgica. Apresentou evolução favorável com atingimento de critérios de cura em 11/2020. Após remoção de cânula de traqueostomia, teve alta para unidade de reabilitação de média duração a 18/01/2021, sem aporte suplementar de oxigénio e com pesquisa de SARS-CoV-2 negativo.

No dia 21/01 encontrava-se polipneica em ar ambiente, SpO2 de 94\%. Exibia respiração toracoabdominal com amplitude torácica residual, simétrica e regular com dificuldade no controlo ventilatório e cansaço fácil a mínimos esforços. Verificou-se diminuição da força muscular sobretudo a nível dos membros inferiores. Capaz de executar levante com ajuda bilateral com potencial para realizar carga e treino de marcha. Equilíbrio estático e dinâmico sentada mantido. Sem capacidade para adoção da posição ortostática com equilíbrio estático ou dinâmico. Necessidade de ajuda parcial nas AVD, exceto na alimentação que é independente e sem défices de deglutição.

Com base na linguagem da CIPE $®$ e no Padrão Documental dos Cuidados de Enfermagem da Especialidade de Enfermagem de Reabilitação, foram identificados os diagnósticos de "ventilação comprometida" e "intolerância à atividade", cujas intervenções que contemplam o programa de RR se encontram direcionadas às necessidades major da pessoa.

Tabela 4 - Enunciado de diagnósticos e intervenções de enfermagem

\begin{tabular}{|c|c|}
\hline \multicolumn{2}{|c|}{ Diagnóstico de Enfermagem: Intolerância à atividade } \\
\hline $\begin{array}{l}\text { Intervenções } \\
\text { de } \\
\text { Enfermagem }\end{array}$ & $\begin{array}{l}\text { - Avaliar intolerância ao esforço [Escala de Borg modificada antes, durante e depois da sessão] } \\
\text { - Planear atividade física [planear o tipo de exercícios e número das repetiçães com a pessoa] } \\
\text { - Gerir atividade física [coordenar com a pessoa fornecendo diretrizes de como realizar o exercício } \\
\text { e quando parar segundo certos sintomas e/ou alteração dos parâmetros vitais] } \\
\text { - Negociar atividade física } \\
\text { - Supervisionar resposta ao exercício [Teste de marcha de } 6 \text { minutos; Treino intervalado (aeróbio): } \\
\text { Exercícios de agachamento com e sem apoio do andarilho; Exercícios ativos livres em posição } \\
\text { ortostática com apoio em barra modificada - flexão coxofemoral e joelho, flexão do joelho, } \\
\text { dorsiflexão plantar, abdução/adução da coxofemoral; Exercícios de equilíbrio e coordenação] } \\
\text { - Instruir sobre técnicas de conservação de energia [ênfase expiratório sincronizado com esforço; } \\
\text { posição de cocheiro quando aumento do cansaço; andar: controlar a respiração e abrandar o } \\
\text { ritmo. Inspirar primeiro e dar alguns passos enquanto expira lentamente; higiene: banho } \\
\text { sentado; vestir-se: dispor a roupa por ordem, preferir roupas largas e elásticas, vestir primeiro a } \\
\text { metade inferior do corpo (sentado), depois a metade superior; dividir o tempo para a realização } \\
\text { de tarefas; evitar movimentos acima dos ombros] }\end{array}$ \\
\hline \multicolumn{2}{|c|}{ Diagnóstico de Enfermagem: Ventilação Comprometida } \\
\hline $\begin{array}{l}\text { Intervenções } \\
\text { de } \\
\text { Enfermagem }\end{array}$ & $\begin{array}{l}\text { - Vigiar a consciência } \\
\text { - Auscultar Tórax } \\
\text { - Observar Tórax } \\
\text { - Vigiar respiração } \\
\text { - Vigiar ventilação } \\
\text { - Monitorizar [saturações periféricas de oxigénio, tensão arterial e frequência cardíaca, no início e } \\
\text { fim da sessão] } \\
\text { - Executar cinesioterapia respiratória [Controlo de dissociação dos tempos respiratórios; Expiração } \\
\text { com lábios semicerrados; Reeducação abdominodiafragmática global sentada; Reeducação costal } \\
\text { global e seletiva, com abertura costal (abdução/adução e flexão/extensão da escapulo umeral, } \\
\text { flexão lateral do tronco) com e sem resistência de } 0,3 \text { kg; Treino com inspirómetro de incentivo } \\
\text { a volume] } \\
\text { - Executar de técnica de posicionamento [posição de descanso e relaxamento sentada, correção } \\
\text { postural] }\end{array}$ \\
\hline
\end{tabular}


Os critérios de prescrição de exercício foram definidos segundo o formato $\mathrm{FITT}^{3,5,17,23}$ (tabela 5), com base no estado de saúde geral da pessoa e nos objetivos a atingir.

Tabela 5 - Prescrição de exercício físico

\begin{tabular}{|c|l|}
\hline Frequência & $3 \times$ por semana \\
\hline Intensidade & Escala de Borg modificada 4 a 6 \\
\hline Tempo & Sessão de 60 minutos \\
\hline Tipo de treino & $\begin{array}{l}\text { Exercícios de reabilitação respiratória } \\
\text { Força [2 a } 3 \text { series de } 10 \text { a } 12 \text { repetições] } \\
\text { Resistência baixa intensidade [6 a } 10 \text { minutos] }\end{array}$ \\
\hline
\end{tabular}

O programa foi implementado de dia 21 de janeiro de 2021 a 4 de março de 2021, compreendendo um total de 14 sessões.

Antes e após a sessão de RR e o TM6 foram avaliados sinais vitais (SpO2, frequência cardíaca e tensão arterial). A inspirometria de incentivo foi realizada sempre após sessão de RR.

Conseguiu-se implementar um treino de baixa intensidade, para obtenção de resultados na redução da sintomatologia, aumento da qualidade de vida e melhoria do desempenho nas AVD; um treino de força com gradual incremento de resistência, centrado nos bíceps, tríceps, deltoides, peitorais e músculos anti gravíticos, promovendo o aumento de carga de cada grupo muscular com o aumento das repetições em cada série; e um treino intervalado no qual se alternou períodos de exercício com períodos de pausa de 2 minutos com benefícios na resposta ventilatória e tolerância ao exercício, seccionados em vários momentos, por esta ordem: exercícios de RR; exercícios de fortalecimento muscular conjugado com técnicas de conservação de energia e controlo ventilatório; treino de equilibro e marcha, e treino com inspirómetro de incentivo a volume.

\section{RESULTADOS}

A colocação em prática deste plano de RR fundamentado em exercícios respiratórios, treino aeróbico e ensinos para a alta induziu na pessoa alterações satisfatórias na avaliação do seu processo patológico e corporal que foram sendo percecionadas e verbalizadas pela própria.

Com base no plano de reabilitação implementado, foi possível constatar ganhos em saúde sensíveis aos cuidados de ER, através da evolução satisfatória dos resultados obtidos com a TM6 e capacidade vital verificando-se em simultâneo a melhoria da perceção subjetiva da tolerância ao esforço.

A fadiga, foi avaliada após ter sido realizada uma apresentação e capacitação da pessoa para a utilização da escala de Borg Modificada. No decorrer das sessões verificou-se uma tendência decrescente na perceção de esforço após atividade, com consequente aumento da tolerância à atividade, mantendo-se sobreponível a sensação de fadiga em repouso.

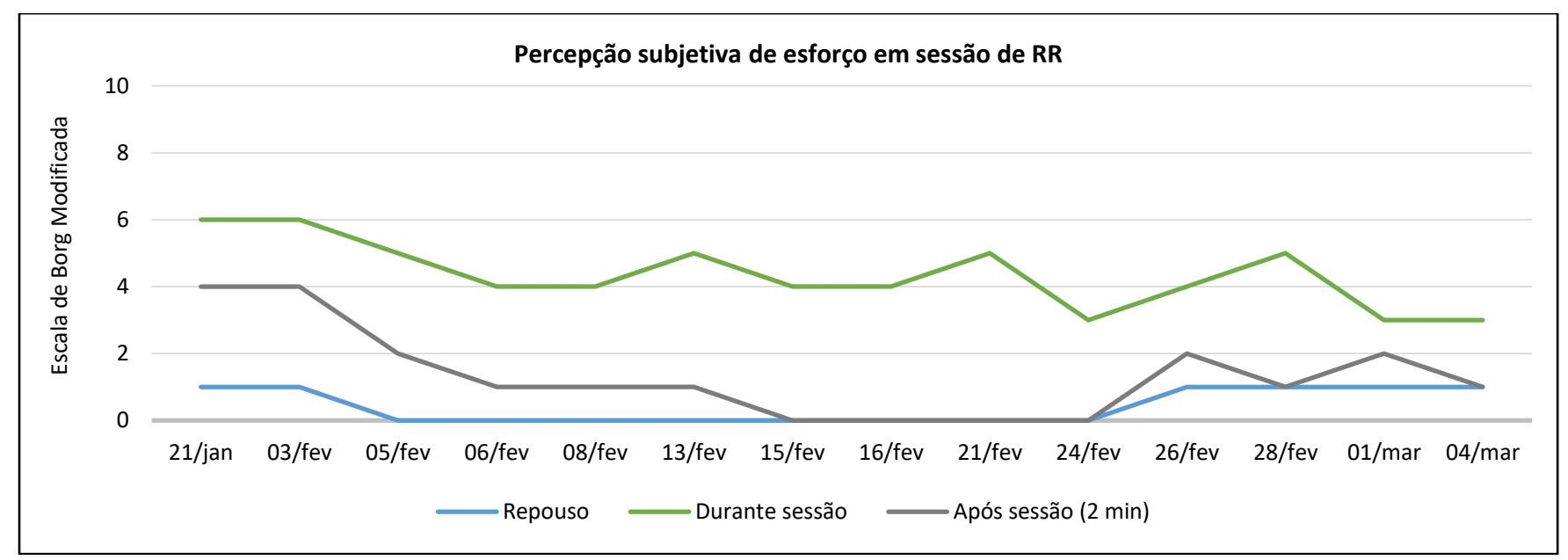

Gráfico 1 - Perceção subjetiva de esforço em sessão de RR

Os critérios de interrupção do exercício serviram para gerir os exercícios implementados de acordo com a tolerância demonstrada, apesar de se ter verificado uma avaliação máxima de 6/10 durante a $1^{\text {a }}$ sessão (gráfico 1), este aspeto foi contornado através do aumento do tempo de pausa entre exercícios e aplicação de estratégias de controlo da respiração. Os valores máximos obtidos na escala de Borg Modificada, em atividade, coincidiram com o 
aumento da utilização da musculatura acessória (quadro 1), principalmente supra-clavicular, beneficiando com os exercícios de descanso e relaxamento sentada.

$\mathrm{Na}$ avaliação do processo corporal abordou-se essencialmente pela inspeção e auscultação pulmonar, ficando aquém da avaliação sugerida pela bibliografia, mas suficiente para a avaliação e complementação dos sinais de fadiga.

Quadro 1 - Resultados da avaliação por inspeção

\begin{tabular}{|c|c|c|c|c|c|}
\hline Data (2021) & Oxigénio (1/min) & Padrão Respiratório & Ritmo & Amplitude & Músculos Acessórios \\
\hline $21 / 01$ & - & Mista & Regular & Superficial & Sim \\
\hline $03 / 02$ & 2 & Torácica & Regular & Superficial & Sim \\
\hline $05 / 02$ & 2 & Torácica & Regular & Superficial & Não \\
\hline $06 / 02$ & 2 & Torácica & Regular & Superficial & Não \\
\hline $08 / 02$ & 1 & Torácica & Regular & Superficial & Não \\
\hline $13 / 02$ & 1 & Torácica & Regular & Superficial & Não \\
\hline $15 / 02$ & 1 & Torácica & Regular & Normal & Não \\
\hline $16 / 02$ & 1 & Mista & Regular & Normal & Não \\
\hline $21 / 02$ & - & Mista & Regular & Normal & Não \\
\hline $24 / 02$ & - & Mista & Regular & Normal & Não \\
\hline $26 / 02$ & - & Mista & Regular & Normal & Não \\
\hline $28 / 02$ & - & Mista & Regular & Normal & Sim \\
\hline $01 / 03$ & - & Mista & Regular & Normal & Não \\
\hline 04/03 & - & Mista & Regular & Normal & Não \\
\hline
\end{tabular}

Não se verificaram assimetrias torácicas, contudo, o padrão respiratório assumiu um predomínio torácico inicialmente com fraca expansão/ amplitude, coincidindo com o período sob aporte de oxigénio. Mantendo a necessidade de intervir com técnicas de reeducação costal global e seletiva com aberturas costais e inspirometria de incentivo por volume, conseguiu-se suspender a oxigenoterapia e diminuir as consequências da rigidez torácica provocadas pela ventilação mecânica invasiva e curarização prolongada e pela imobilidade no leito (quadro 1).

Quadro 2 - Resultados da avaliação por auscultação

\begin{tabular}{|c|c|c|}
\hline Data (2021) & Murmúrio vesicular & Ruídos Adventícios \\
\hline $21 / 01$ & $\downarrow$ globalmente & Não \\
\hline $03 / 02$ & $\downarrow$ globalmente \\
\hline $05 / 02$ & $\downarrow$ globalmente & Fervores Crepitantes \\
\hline $06 / 02$ & $\downarrow$ globalmente & Fervores Crepitantes \\
\hline $08 / 02$ & $\downarrow$ globalmente & Fervores Crepitantes \\
\hline $13 / 02$ & $\downarrow$ globalmente & Fervores Crepitantes \\
\hline $15 / 02$ & Presente & Fervores Crepitantes \\
\hline $16 / 02$ & Presente & Fervores Crepitantes \\
\hline $21 / 02$ & Presente & Fervores Crepitantes \\
\hline $24 / 02$ & Presente & Fervores Crepitantes \\
\hline $26 / 02$ & Presente & Fervores Crepitantes \\
\hline $28 / 02$ & Presente & Fervores Crepitantes \\
\hline $01 / 03$ & Presente & Fervores Crepitantes \\
\hline $04 / 03$ & Presente & Fervores Crepitantes \\
\hline
\end{tabular}

Relativamente à auscultação (quadro 2), comprovou-se a presença de murmúrio vesicular diminuído em ambos os campos pulmonares acompanhados de redução do tempo inspiratório, justificado pela patologia pulmonar crónica 
restritiva provocada pela COVID-19. A evidência de ruídos adventícios na fase inspiratória e permanentes nas bases, é corroborada pela possível condensação pulmonar localizada, condicionando o colapso dos alvéolos existentes em maior número.

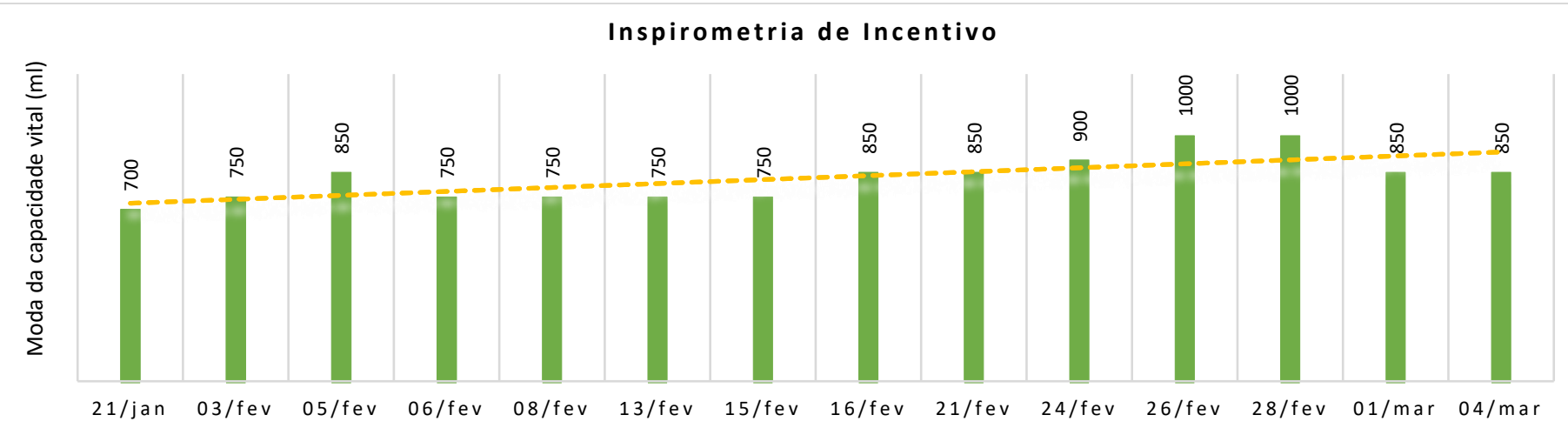

Gráfico 2 - Inspirometria de incentivo após sessão de RR

A inspirometria de incentivo por volume foi realizada mediante ensino prévio ainda em meio hospitalar.

Nas patologias restritivas, o problema primário é a perda de volume pulmonar; assim, o achado fundamental é uma redução na capacidade pulmonar total (CPT). Isso é determinado por medidas diretas, ou seja, uma CV reduzida, na medida em os músculos inspiratórios fracos limitam o volume de uma inspiração máxima, enquanto os músculos expiratórios fracos evitam o esvaziamento completo.

O feedback visual potenciado pelo inspirómetro de incentivo encorajou a execução da inspiração máxima sustentada, verificando-se uma linha de tendência ascendente da CV ao longo das sessões, o que se traduziu numa melhoria da força muscular respiratória, função pulmonar e mobilidade toracoabdominal imprimida pelos exercícios de reabilitação respiratória realizados e constatada na tolerância aos exercícios aeróbios e de força.

Quadro 3 - Avaliação de parâmetros vitais em repouso e após TM6

\begin{tabular}{|c|c|c|c|c|c|c|c|c|c|}
\hline & Datas (2021) & $15 / 02$ & $16 / 02$ & $21 / 02$ & $24 / 02$ & $26 / 02$ & $28 / 02$ & $01 / 03$ & $04 / 03$ \\
\hline \multirow{3}{*}{ 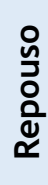 } & Tensão Arterial (mmHg) & $153 / 72$ & $144 / 82$ & $142 / 73$ & $140 / 66$ & $141 / 72$ & $141 / 68$ & $144 / 72$ & $139 / 73$ \\
\hline & Frequência Cardíaca (bpm) & 72 & 70 & 75 & 79 & 87 & 80 & 77 & 82 \\
\hline & $\mathrm{SpO} 2(\%)$ & 98 & 98 & 97 & 94 & 95 & 90 & 91 & 92 \\
\hline \multirow{3}{*}{ 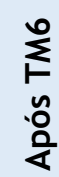 } & Tensão Arterial (mmHg) & $176 / 81$ & $177 / 87$ & $174 / 78$ & $145 / 73$ & $184 / 93$ & $178 / 84$ & $173 / 80$ & $151 / 73$ \\
\hline & Frequência Cardíaca (bpm) & 78 & 77 & 76 & 84 & 98 & 94 & 84 & 97 \\
\hline & SpO2 (\%) & 91 & 93 & 94 & 93 & 80 & 80 & 75 & 85 \\
\hline
\end{tabular}

Os parâmetros vitais foram avaliados na sequência da realização do TM6 com o intuito de determinar a resposta ao tratamento instituído, no que diz respeito à tolerância à atividade. No primeiro dia foi realizado treino apesar dos valores de tensão arterial tendo sido bem tolerado pela pessoa.

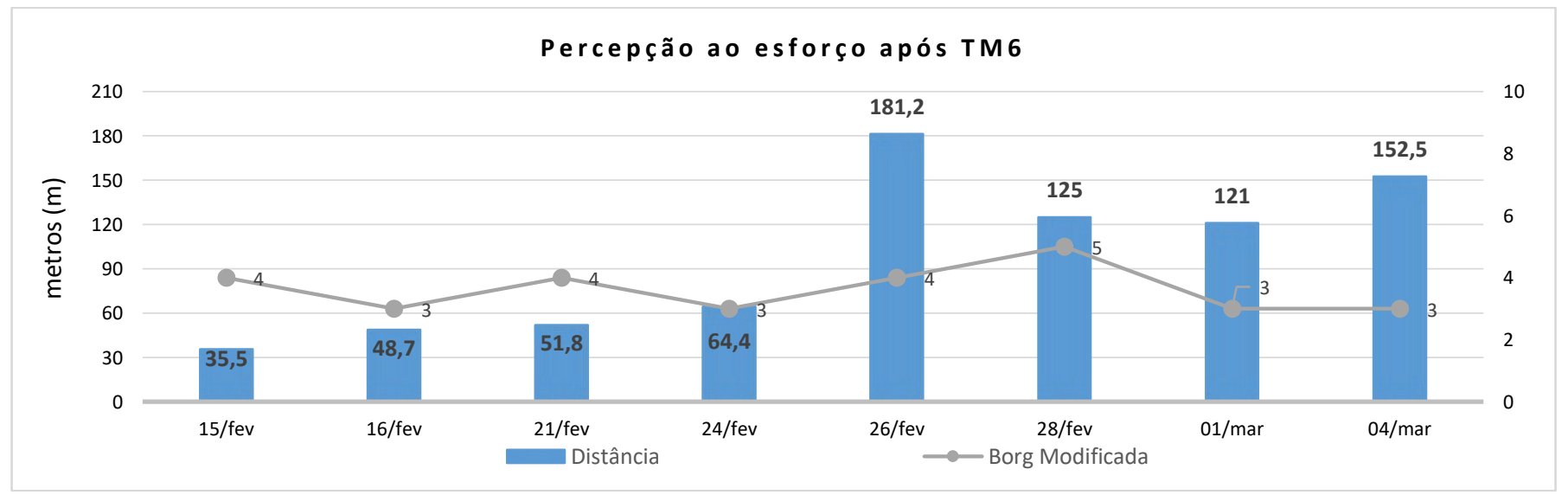

Gráfico 3 - Perceção subjetiva ao esforço após TM6 
Constatou-se um aumento progressivo da distância percorrida sem prejuízo nos valores relativos à tensão arterial e frequência cardíaca, demonstrando-se estáveis dentro dos critérios definidos previamente. Contudo os valores das oximetrias periféricas (quadro 3) não foram relacionados com esse fato nem comprometeram o plano de cuidados, pois a avaliação encontrou-se condicionada pela alteração da perfusão periférica com presença de extremidades frias, não tendo sido valorizada pela ausência de outros sinais e sintomas de dessaturação.

As alterações mais significativas corresponderam ao relato de uma avaliação de 5 em 10 na escala de Borg Modificada relacionado com o aumento do ritmo de passada, sem incentivo para tal, com necessidade de pausa no teste ao $4^{\circ}$ minuto. No teste implementado foi aferida uma redução e/ou controlo da fadiga, revelada pelos valores estáveis de perceção do esforço apresentados no gráfico 3 e com consequente melhoria das limitações às AVD, com ganho de independência progressiva no autocuidado de higiene, uso do sanitário, vestuário, arranjar-se, e andar com auxiliar de marcha.

Em suma, capacidade para o exercício melhorou após programa de RR, quando inferida pela melhoria TM6, achado que se correlaciona com os valores de CV.

\section{DISCUSSÃO}

A apresentação deste caso demonstra que a RR assume importância como medida não farmacológica complementar no tratamento de pessoas com sequelas pós-COVID, mais estritamente na sintomatologia sequelar apresentada.

A reabilitação sendo a disciplina que visa melhorar a funcionalidade assume um papel relevante na resposta a este indicador, sendo possível reduzir o declínio funcional e acelerar a recuperação em situações agudas ou crónicas através da $\mathrm{RR}^{18}$. Estes programas desempenham um papel benéfico no aumento da tolerância ao esforço, recuperação da independência, melhoria da qualidade de vida com impacto nas AVD ${ }^{3,17}$.

Os exercícios de técnicas respiratórias como controlo e dissociação dos tempos respiratórios com lábios semicerrados; inspiração profunda e sustentada associada a expiração com resistência, relaxamento, exercícios abdominodiafragmáticos, exercícios de expansão torácica e abertura costal, com evidência científica significativa na melhoria da função respiratória, mobilidade torácica, ganho de força, resistência e na reinserção da pessoa nas suas $\mathrm{AVD}^{4,5,6,16}$, foram adequados e bem tolerados confirmando as conclusões de outros trabalhos publicados. 0 treino aeróbio e exercício de força muscular na mesma perspetiva, são sugeridos como domínios integrantes do plano de reabilitação pós-covid com foco na melhoria sintomatológica e da dinâmica ventilatória, e com benefícios na capacidade funcional e descondicionamento físico ${ }^{1,9,18,24}$.

A aplicação da Escala de Borg Modificada como instrumento de avaliação demonstrou-se importante para este estudo pelo foco da questão norteadora e pela importância atribuída pelas orientações da mesa do colégio da especialidade de ER, mostrando-se sensíveis à fadiga percecionada pela pessoa na realização dos exercícios e no controlo e sustentação do treino aeróbio. Estudos referem a utilização de máscara cirúrgica como possível enviesamento de resultados, pois poderá estar na origem do desconforto e redução do desempenho com consequente deturpação da perceção do esforço ${ }^{18}$.

Verificou-se uma diminuição da fadiga em atividade e após recuperação, apesar de não existir espaço temporal suficiente para afirmar uma redução permanente. A monitorização das saturações periféricas de oxigénio, frequência cardíaca e escala de Borg Modificada conferiu segurança às sessões descartando critérios de interrupção do exercício, como comprova Morales ${ }^{25}$.

Na prescrição de exercício e na avaliação da capacidade funcional em doentes pós COVID-19 é unanime a utilização do TM6 como forma de identificar a resposta individual ao plano instituído e avaliar a hipoxémia silensiosa ${ }^{18}$. Fatores que podem ter influenciado negativamente este teste são a fraqueza dos músculos pélvicos e quadricípites ${ }^{12,16}$, a baixa estatura, o género feminino, a idade avançada e o estado de saúde comprometido ${ }^{25}$.

A corroborar os resultados do presente estudo, Cacau ${ }^{16}$ refere evidência científica de estudos cujo resultado de distância percorrida do TM6 foi inferior a 200 metros em doentes pós-COVID; Moreira ${ }^{26}$ relata que após o programa de reabilitação a tolerância à atividade melhora se inferida pelo teste de caminhada de 6 minutos e relaciona ainda a distância percorrida com a fraqueza dos quadricípites; Ferreira ${ }^{18}$ avança descrevendo que mesmo 5 anos pósARDS nem sempre atingem a distância padronizada.

Os critérios de inclusão definidos pela diversa bibliografia mostraram-se adequados e ajustados a uma prática segura, contudo as alterações neurocirculatórias das extremidades constituíram um obstáculo ao estudo, inviabilizando a avaliação das oximetrias periféricas e sua correlação com outros dados. Os obstáculos na realização do TM6 foram superados com a contabilização da distância total percorrida através da medição a laser do corredor. Para manutenção do ambiente seguro a pessoa manteve-se sempre acompanhada e com auxílio de dispositivo auxiliar de marcha (andarilho) para diminuição da ansiedade e tensão muscular provocada pelo medo de queda.

Este estudo veio contribuir para o desenvolvimento do conhecimento na capacitação da pessoa com deficiência, limitação da atividade e/ou restrição da participação, para a sua reinserção, bem como na maximização da sua funcionalidade. 
A reabilitação deve ser guiada com uma atitude rigorosa e baseada em evidências, requerendo uma pesquisa bibliográfica direcionada para o objetivo traçado. Atualmente existem dezenas de exercícios respiratórios, programas de treino, e até mesmo consenso de especialistas e diretrizes, mas os estudos exploratórios são muito pouco conclusivos, deste modo este estudo vem apresentar um caso de um plano de RR em doente pós-Covid com dois focos de diagnóstico prementes para a intervenção do EEER nesta tipologia de doentes.

É um caso estimulante pela sua atualidade, que requereu muita pesquisa para otimização do programa de reabilitação implementado podendo dotar a pessoa de informação fidedigna e potenciar alicerces à educação. Contudo, a evidência que sustenta o plano instituído, apesar do exponencial surgimento de bibliografia referente aos cuidados de reabilitação nestes caos, é ainda reduzida no que concerne ao seu benefício e em que momento da fase da doença.

\section{CONCLUSÃO}

Este estudo de caso permitiu verificar a efetividade das intervenções do EEER na implementação de um plano de intervenção numa pessoa portadora de sequelas da COVID-19 direcionado para o controlo e redução da fadiga.

Em resposta aos objetivos enunciados foi possível determinar o contributo do EEER no processo de recuperação da pessoa com sequelas de COVID-19, nomeadamente no controlo e redução da fadiga com evidentes ganhos em saúde. Foi possível determinar uma diminuição/normalização da perceção de cansaço ao esforço com aumento progressivo da distância percorrida durante o TM6, melhoria da amplitude torácica e aumento da capacidade vital pulmonar, remoção do suporte de oxigenoterapia, e com melhoria da autonomia da pessoa para as AVD.

Pretende-se que os EEER repliquem mais programas de reabilitação direcionados para estes doentes, identificando os ganhos sensíveis aos cuidados de enfermagem de reabilitação.

\section{REFERÊNCIAS BILIOGRÁFICAS}

1. Agostini F, Mangone M, Ruiu P, Paolucci T, Santilli V, Brernetti A. Rehabilitation settings during and after covid-19: an overview of recommendations. J Rehabil Med. 2021; 53.

2. Fernandes PM, Mariani AW. Life post-COVID-19: symptoms and chronic complications [editorial]. São Paulo Med J. 2021 ; $139(1)$ : 1 -2. https://doi.org/10.1590/1516-3180.2021.139104022021

3. Silva LC, Pina TA, Ormond LS. Sequelas e reabilitação pós-covid19: revisão de literatura. Revista das Ciências da Saúde e Ciências aplicadas do Oeste Baiano -Higia. 2021; 6(1): 169-184.

4. Nielsen CC, Silva CC. Reabilitação pulmonar em pacientes após covid-19: uma proposta. Biblioteca Digital do Exército. 2020 Nov.

5. Cuidados de enfermagem de Reabilitação para pessoas com COVID-19. ORIENTAÇÕES - COVID-19. Mesa do colégio da especialidade de enfermagem de reabilitação. 2020.

6. Jin YH, Cai L, Cheng ZS, Cheng H, Deng T, Fan YP et al. A rapid advice guideline for the diagnosis and treatment of 2019 novel coronavirus (2019-nCoV) infected pneumonia (standard version). Military Medical Research. 2020; 7:4. https://doi.org/10.1186/s40779-020-0233-6

7. Yang F, Liu N, Wu JY, Hu LL, Su GS, Zheng NS. Pulmonary rehabilitation guidelines in the principle of 4 S for patients infected with 2019 novel coronavirus (2019-nCoV). Zhonghua Jie He He Hu Xi Za Zhi. 2020; 43(0):E004. https://doi.org/10.3760/cma.j.issn.10010939.2020.03.007

8. Matos AP, Dias JL, Sardinha J. COVID-19: Relato Radiológico de Dois Casos. Gazeta. 2020; 7 (2): 208-212.

9. Brugliera L, Spina A, Castellazzi P, Cimino P, Tettamanti A, Houdayer E, et al. Rehabilitation of covid-19 patients [Letter to the editor]. J Rehabil Med. 2020; 52: jrm00046. https://doi.org/10.2340/16501977-2678

10. Garg P, Arora U, Kumar A, Malhotra A, Kumar S, Garg S et al. Risk factors for prolonged fatigue after recovery from COVID-19. J Med Virol. 2021; 93: 1926-1928. https://doi.org/10.1002/jmv.26774

11. Jianam LI. Rehabilitation management of patients with COVID-19: lessons learned from the first experience in China. European Journal of Physical and Rehabilitation Medicine. 2020; 56(3): 335-338. https://doi.org/10.23736/S1973-9087.20.06292-9

12. Chérrez-Ojeda I, Gochicoa-Rangel L, Salles-Rojas A, Mautong H. Follow-up of patients after COVID-19 pneumonia. Rev Alerg Mex. 2020; 67(4): 350-369. https://doi.org/10.29262/ram.v67i4.847

13. O'Sullivan O. Long-term sequelae following previous coronavirus epidemics. Royal College of Phtsicians. 2021: 21(1): e68-70. https://doi.org/10.7861/clinmed.2020-0204

14. Tenforde MW, Kim SS, Lindsell CJ, Billig Rose E, Shapiro NI, Files DC, et al. Symptom Duration and Risk Factors for Delayed Return to Usual Health Among Outpatients with COVID-19 in a Multistate Health Care Systems Network - United States. Morb Mortal Wkly Rep. 2020; 69(30):993-998. https://doi.org/10.15585/mmwr.mm6930e1

15. Nalbandian, A., Sehgal, K., Gupta, A. et al. Post-acute COVID-19 syndrome. Nat Med 27, 601-615 (2021). https://doi.org/10.1038/s41591021-01283-z

16. Cacau LA, Mesquita R, Furlanetto KC, Borges DL, Forgiarni JR. LA, Maldaner V et al. Avaliação e intervenção para a reabilitação cardiopulmonar de pacientes recuperados da COVID-19. ASSOBRAFIR Ciência. 2020; 11(1): 183-193. http://dx.doi.org/10.47066/21779333.AC20.covid19.018

17. Mesa do Colégio de Enfermagem de Reabilitação. Guia Orientador de Boa Prática - Reabilitação Respiratória. Lisboa: Ordem dos Enfermeiros; 2018.

18. Ferreira BF, Tozato C, Molinarl CV, Papa V, Guizilini S, Ferreira VM et al. Reabilitação cardiopulmonar na covid-19. Rev Soc Cardiol Estado de São Paulo. 2020; 30 Supl 4: 531-536. http://dx.doi.org/10.29381/0103-8559/20203004531-6

19. Andrade SR, Ruoff AB, Piccoli T, Schmitt MD, Ferreira A, Xavier AC. O estudo de caso como método de pesquisa em enfermagem: uma revisão integrativa. Texto Contexto Enferm. 2017; 26(4): e5360016. http://dx.doi.org/10.1590/0104-07072017005360016

20. Silva R, Carvalho A, Rebelo L, Pinho N, Araújo T, Ribeiro 0 et al. Contributos do referencial teórico de Afaf Meleis para a Enfermagem de 
Reabilitação. Revista de Investigação em Enfermagem. 2019: 35 - 44. https://www.researchgate.net/publication/337313131

21. Chinese Association of Chest Physician; Respiratory Rehabilitation Committee of Chinese Association of Rehabilitation Medicine. Recommendation of respiratory rehabilitation for PICS in critically ill patients with COVID-19. Zhonghua Jie He He Hu Xi Za Zhi. 2020; 43(9):737-743. http://dx.doi.org/10.3760/cma.j.cn112147-20200512-00592

22. Zhao HM, Xie YX, Wang C. Recommendations for respiratory rehabilitation in adults with coronavirus disease 2019. Chinese Medical Journal. 2020; 133(13): 1595-1602. https://dx.doi.org/10.1097/CM9.0000000000000848

23. Comitiva de Trabalho de Reabilitação Respiratória. Recomendações para a Retoma de Atividade das Unidades de Reabilitação Respiratória durante a fase de mitigação de infeção COVID-19. Sociedade Portuguesa de Pneumologia 2020.

24. Programa Nacional de Prevenção e Controlo de Infeções e das Resistências aos Antimicrobianos da Direção-Geral da Saúde. Prevenção e Controlo de Infeção por SARS-CoV-2 (COVID-19): Equipamentos de Proteção Individual (EPI). Norma Nº07/2020, (29/03/2020).

25. Morales-Blanhir JE, Vidal CD, Romero MJ, Castro MM, Villegas AL, Zamboni M. Teste de caminhada de seis minutos: uma ferramenta valiosa na avaliação do comprometimento pulmonar. J Bras Pneumol. 2011; 37(1): 110-117. https://doi.org/10.1590/S1806-37132011000100016

26. Moreira MA, Moraes MR, Tannus R. Teste da caminhada de seis minutos em pacientes com DPOC durante programa de reabilitação. J Pneumol. 2001; 27(4): 295-300. https://doi.org/10.1590/S0102-35862001000600002

\section{DIVULGAÇÕES ÉTICAS}

\section{Contribuição do(s) autor(es):}

Conceptualização: FB e HP

Metodologia: FB; AM; PF e HP

Validação: $F B ; A M ; P F$ e HP

Análise formal: FB; $A M$; PF e HP

Investigação: FB; PF e HP

Tratamento de dados: FB e HP

Preparação do rascunho original: FB; PF e HP

Redação e edição: $\mathrm{FB}$ e HP

Revisão: FB; AM; PF e HP

Todos os autores leram e concordaram com a versão publicada do manuscrito.

Financiamento:

Este trabalho não recebeu nenhuma contribuição financeira ou bolsa.

Declaração de consentimento informado:

0 consentimento informado por escrito para publicar este trabalho foi obtido dos participantes.

Conflitos de interesse:

Os autores não declaram nenhum conflito de interesses.

(c) Autor (es) (ou seu (s) empregador (es)) e APER/RPER 2022. Reutilização permitida de acordo com CC BY-NC-ND. Nenhuma reutilização comercial. 